\title{
Autoimmune Thrombocytopenia Complicated by EDTA- and/or Citrate-Dependent Pseudothrombocytopenia
}

\author{
Abdulgabar Salama \\ Institute for Transfusion Medicine, Charité - Universitätsmedizin Berlin, Berlin, Germany
}

\section{Keywords}

Pseudothrombocytopenia - ITP . EDTA-dependent .

Citrate-dependent $\cdot$ Heparin-dependent

\section{Summary}

Background: Pseudothrombocytopenia (PTCP) is a wellknown phenomenon. However, confusion may occur due to unusual characteristics. Case Reports: Two patients with autoimmune thrombocytopenia (ITP) and long-lasting PTCP are described. Initially, only the diagnosis of ITP was confirmed. During observation, discrepancies were recognized between clinical findings and platelet counts. Re-examination resulted in the additional diagnosis of EDTA-dependent PTCP. Subsequently, the latter diagnosis was changed to citratedependent PTCP in both cases. Interestingly, PTCP was observed to change again and became recognizable in citrate or heparin, and only during the first 20-30 min following phlebotomy in EDTA specimens. Conclusion: The incidence of concomitant ITP with PTCP might be higher than previously reported, and PTCP may have variable dynamics and characteristics.

(c) 2015 S. Karger GmbH, Freiburg

\section{Introduction}

Pseudothrombocytopenia (PTCP) is a well-known phenomenon which is most commonly reflected by clumping of platelets in EDTA and rarely in citrate, or in the presence of IgM autoantibodies to platelets [1-4]. Its pathogenesis, incidence, and association with diseases have been extensively discussed [5-8].
Though the phenomenon per se is harmless, its occurrence may lead to confusion and inadequate interventions including extensive laboratory testing and/or diagnostics, false diagnosis, and consequently false treatment [9-15]. True thrombocytopenia might also rarely be confused [15] or associated with PTCP [16-18]. Recently, PTCP has also been described in a patient receiving romiplostim for ITP [19]. Here, we report on long-lasting PTCP complicated by differential characteristics in 2 patients with ITP.

\section{Case Reports}

\section{Case 1}

A 55-year-old women with a long-term history of psoriasis, thrombocytopenia (platelet counts ranging between $19,000 / \mu \mathrm{l}$ and $99.000 / \mu \mathrm{l}$ ), and an infrequent bleeding tendency was admitted to the hospital in the year 2000 due to ITP and recurrent infections. IgG autoantibodies to the platelet glycoprotein $\mathrm{IIb} / \mathrm{III}$ a were detected on patients' platelets by MAIPA (monoclonal antibodyspecific immobilization of platelet antigens), but not in the serum. In addition, a third diagnosis of IgG deficiency was confirmed. The patient was under treatment with low doses of prednisolone, and sulfasalazine for psoriatic arthritis. During observation, sulfasalazine was replaced by methotrexate and intravenous IgG (IVIgG) treatment which was administered every $6-8$ weeks. In 2002, platelet counts decreased to $2,000 / \mu \mathrm{l}$, although the patient felt well and there was no evidence for any bleeding tendency (table 1). In citrate blood, platelet counts were found to be $\geq 100,000 / \mu \mathrm{l}$, and PTCP related to EDTA was confirmed by microscopy. One year later, the PTCP was found to be associated with citrate rather than EDTA. The platelet count was observed to be infrequently less than $10,000 / \mu \mathrm{l}$, independent of the anticoagulant used. It remained a matter of speculation whether PTCP was abolished or could not be recognized due to the very low platelet counts. It is worth mentioning that platelet counting was immediately performed following phlebotomy at that time. In 2015 we recognized by a chance, time-dependent PTCP. The patient felt well, and there was no evidence for the presence of true thrombocytopenia. In fact, repeated analysis of platelet counts by using the same blood samples revealed an increase from 3,000/ $\mu \mathrm{l}$ to $81,000 / \mu \mathrm{l}$ in EDTA, but not in citrate and heparin (fig. 1).

Case 2

An 18-year-old female, who had been diagnosed with ITP in 2004, was admitted to the hospital in 2009. The patient felt well, and the platelet count was

\section{KARGER}

Fax +497614520714

\section{(c) 2015 S. Karger GmbH, Freiburg}

$1660-3796 / 15 / 0425-0345 \$ 39.50 / 0$ 
Table 1. Course of thrombocytopenia and PTCP in patient no. 1; all blood samples were withdrawn into vacutainers

\begin{tabular}{|c|c|c|c|c|c|c|c|}
\hline \multirow[t]{2}{*}{ Year(s) } & \multicolumn{3}{|c|}{ Pltr $\times 10^{3} / \mu \mathrm{l} /$ aggregates } & \multirow[t]{2}{*}{ Blt } & \multirow[t]{2}{*}{ Diagnosis } & \multirow[t]{2}{*}{ Plab } & \multirow[t]{2}{*}{ Treatment } \\
\hline & EDTA & citrate & heparin & & & & \\
\hline $1964-1999$ & 19-99 / n.t & n.t & n.t & mild & PS, Arth, Thrp & n.t. & Diclo, Pred, Sul, L-Thyr \\
\hline 2000 & $112 /$ n.t & n.t & n.t & mild & Нyp, ITP & GP IIb/IIIa & MTX, Simva, Meto, Rami, L-Thyr \\
\hline 2001 & $2 /+$ & $110 /-$ & n.t & mild & PTCP & & MTX, Simva, Meto, Rami \\
\hline 2002 & $2-85 /+$ & $>100 /-$ & n.t & - & & & \\
\hline 2004 & $40-86 /(+)$ & $2-19 /+$ & n.t & - & & $\operatorname{IgM}, \operatorname{IgG}^{* * *}$ & \\
\hline 2005 & $2-96 /(+)$ & $2-30 /+$ & n.t & - & $\mathrm{BrCa}$ & & MTX, IVIgG, Simva, L-Thyr, Anast, Ena \\
\hline 2006 & $77-90 /(+)$ & n.t & n.t & mild & & & \\
\hline 2007 & $2-126 /+$ & $<10 /+$ & $<10 /+$ & - & & & MTX, IVIgG, Simva, L-Thyr, Ena, Verap \\
\hline 2008 & $18-80 /(+)$ & $<10 /+$ & $<10 /+$ & & & & MTX, Simva, L-Thyr, Ena, Verap, Anast \\
\hline 2009-2014 & $7-82 /(+)$ & n.t & n.t & mild & & & Verap, L-Thyr, Simva, Anast, Neb, HCT \\
\hline $2015^{\star *}$ & $80 /-$ & $<10 /+$ & $<10 /+$ & - & & & Anast, Cand \\
\hline
\end{tabular}

Arth = Arthritis; Anast = anastrozole; Blt = bleeding tendency; Cand = candesartan; Diclo = diclofenac; Ena = enalapril; HTC = hydrochlorothiazide; Hyp = hypertension; Hypo = hypothyreosis; ITP = autoimmune thrombocytopenia; IVIgG = intravenous IgG; L-Thyr = L-thyroxine; Meto = metoprolole; MTX = methotrexate; Neb = Nebivolol; n.t. = not tested or unknown; Plab = platelet antibodies; Pltr = Platelet range; Pred = prednisolone; PS = psoriasis; PTCP = pseudothrombocytopenia; Rami = ramipril; Simva = simvastatine; Sul = sulfasalazine; Thrp = thrombocytopenia; Verap = verapamil; $(+)=$ weak aggregates; + = significant aggregates.

${ }^{*}$ Immediately after phlebotomy.

**30 min after phlebotomy (see fig. 1).

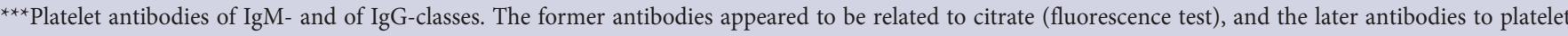
antigens.

Table 2. Course of thrombocytopenia and PTCP in patient no. 2, all blood samples were withdrawn into vacutainers

\begin{tabular}{|c|c|c|c|c|c|c|c|}
\hline \multirow[t]{2}{*}{ Year(s) } & \multicolumn{3}{|c|}{ Pltr $\times 10^{3} / \mu \mathrm{l} /$ aggregates } & \multirow[t]{2}{*}{ Bleeding } & \multirow[t]{2}{*}{ Diagnosis } & \multirow[t]{2}{*}{ Plab } & \multirow[t]{2}{*}{ Treatment } \\
\hline & EDTA & citrate & heparin & & & & \\
\hline 2004-2006 & $<10->50 /$ n.t. & n.t. & n.t. & + & ITP & n.t. & Pred, IVIgG, Cyclo \\
\hline 2007-2008 & $>50$ / n.t. & n.t. & n.t. & - & & n.t. & no treatment \\
\hline 2009 & $<20-61 /$ n.t. & n.t. & n.t. & + & & n.t. & Pred \\
\hline 2010 & $1-24$ / n.t. & n.t. & n.t. & + & & - & Anti-D, Dexa, Pred, Methylpred \\
\hline \multirow[t]{2}{*}{2013} & $<20-32 /+$ & n.t. & n.t. & + & + AIHA & - & Dexa, Pred \\
\hline & $28-32 /-$ & n.t. & n.t. & - & & GP IIb/IIIa & Pred, Aza \\
\hline \multirow[t]{2}{*}{2014} & $17-44 /-$ & n.t. & & & & & \\
\hline & $5 /$ n.t. & $50 /$ n.t. & $58 /$ n.t. & - & & - & \\
\hline \multirow[t]{2}{*}{2015} & 24 / n.t. & 107 / n.t. & n.t. & - & & & Pred \\
\hline & 85 / n.t. & 13 / n.t. & n.t. & - & & & \\
\hline
\end{tabular}

AIHA = Autoimmune hemolytic anemia; Aza = azathioprine; Cyclo = cyclophosphamide; Dexa = dexamethasone; ITP = autoimmune thrombocytopenia; IVIgG = intravenous immunoglobulins; Methylpred = methyl-prednisolone; n.t. = not tested or unknown; Plab = platelet autoantibodies; Pltr = platelet range; Pred $=$ prednisolone; $+=$ significant aggregates .

observed to be $61,000 / \mu l$ without treatment (table 2). In 2010, a bleeding tendency developed, and the platelet count increased from $4,000 / \mu \mathrm{l}$ to more than $100,000 / \mu$ following treatment. IgG autoantibodies to the platelet glycoprotein IIb/IIIa were detected on patients' platelets by MAIPA, but not in the serum. In 2013, the patient developed autoimmune hemolytic anemia of the warm type (Evans syndrome) and her platelet count increased from $10,000 / \mu l$ to a maximum of 320,000/ $\mu$ following treatment with elthrombopag, whereas low-dose prednisolone and azathioprine remained ineffective. An EDTA-dependent PTCP was unexpectedly detected in 2014. This was observed to change to citrate and time-dependent PTCP in 2015 (fig. 1). It remains unclear whether the thrombocytopenia diagnosed in 2004 is somewhat related to EDTA.

\section{Discussion}

The PTCP described here was rather obscure in regards to various aspects. It was complicated with ITP which is per se characterized by thrombocytopenia. The question whether PTCP was already present prior to the manifestation of ITP or developed during observation independent of ITP remains speculative. However, its long-lasting nature appears to be reflected by an association rather than a coincidence. This question has previously been discussed in 

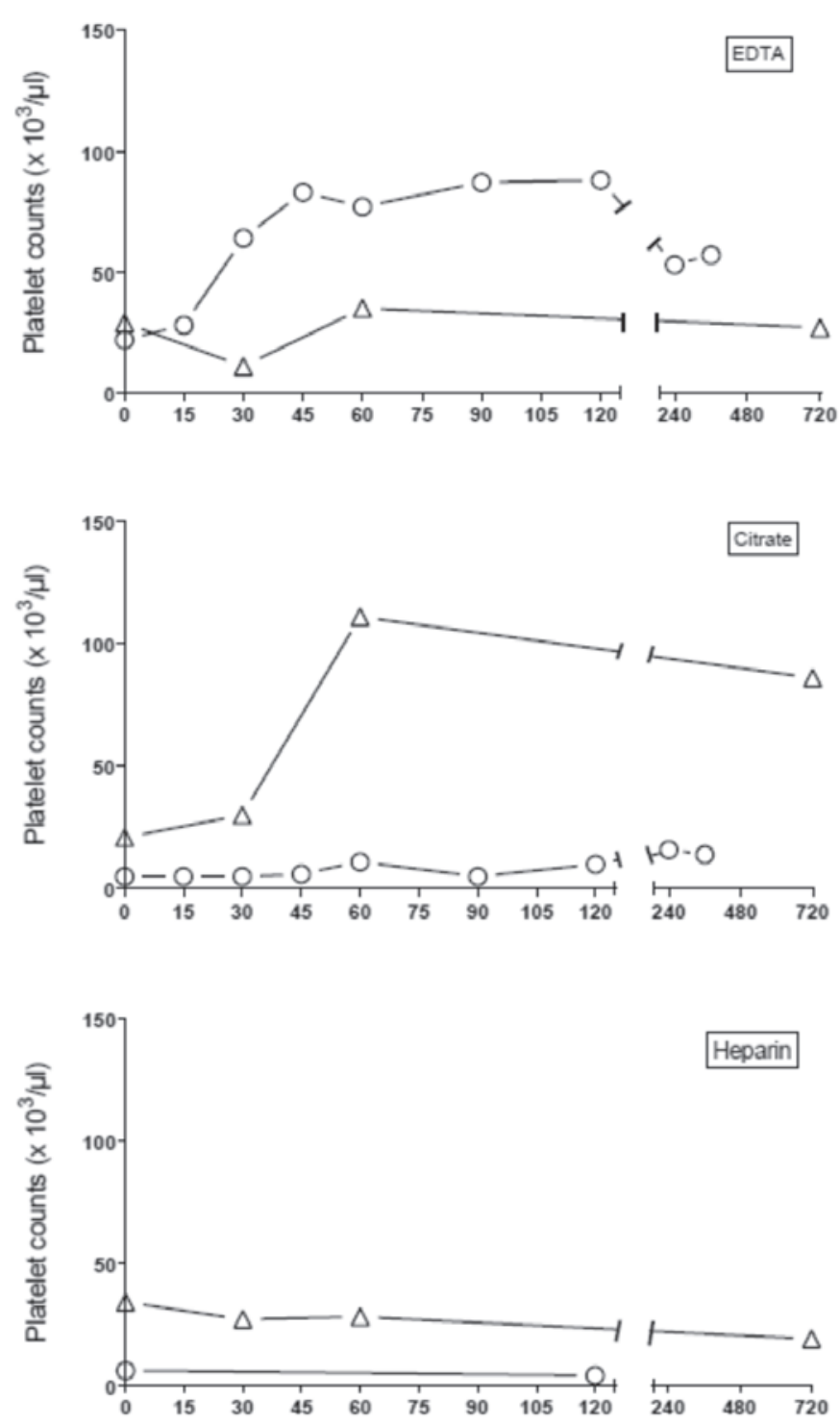

minutes

Fig. 1. Time and anticoagulant-dependent PTCP in 2 patients with ITP $(\mathrm{O}=$ patient no. 1, $\Delta=$ patient no. 2). two patients who were receiving heparin and developed anti-PF4 IgM antibodies. In both cases, platelet aggregation was dependent on the antibodies, resulting in the question of whether the concomitance was reflected by a coincidence or an association [17]. In contrast, a true association could be demonstrated in two other patients who had heparin-induced thrombocytopenia and PTCP [18].

In our patients, PTCP was independent of the true platelet count and ITP. This finding may reflect rather a coincidence than an association. On the other hand, the long-lasting course of both ITP and PTCP do support rather an association than an unexpected coincidence. Another possible explanation might be that the PTCP in the first patient was related rather to the psoriasis than to the ITP. This notion is supported by the clinical course of the patient and by a previous report describing PTCP in a patient with a medical history of benign psoriasis [20]. Though no association was discussed in that report, an association cannot be excluded.

Another strange aspect is the switch from EDTA- to citrate-dependent PTCP in both patients. The mechanism by which this switch may operate remain obscure. For the most part, EDTA is responsible for the vast majority of PTCPs, and citrate is used to disclose this phenomenon. Correspondingly, this was, in fact, initially the case in our patients. The reason for the switch described here remains unclear.

Most intriguingly, the PTCP in case 1 was not only anticoagulant-dependent but also EDTA- and time-dependent. This finding could be proven by repeated platelet counting using identical blood samples. This phenomenon has not yet been described in PTCP. Usually, clumping of platelets in the presence of EDTA is inversely related to the length of time elapsed since phlebotomy $[9,20]$.

We recommend to consider PTCP not only in the list of ITP differential diagnosis but to also include ITP in the list of diseases associated with PTCP. In addition, PTCP may alter its appearance over time. Recently, Nagler et al. [22] have described a typical histogram of PTCP in an EDTA sample as compared to normal histogram in a citrated sample of the same patient. Ultimately the use of CTP (citrate, pyridoxal 5区-phosphate and Tris) as anticoagulant may be helpful in suspected cases.

\section{Disclosure Statement}

The author declares not conflict of interest.

\section{References}

1 Payne BA, Pierre RV: Pseudothrombocytopenia: a laboratory artifact with potentially serious consequences. Mayo Clin Proc 1984;59:123-125

2 Schrezenmeier H, Müller H, Gunsilius E, Heimpel H, Seifried E: Anticoagulant-induced pseudothrombocytopenia and pseudoleucocytosis. Thromb Haemost 1995; 73:506-513.

- 3 Lippi G, Plebani M: EDTA-dependent pseudothrombocytopenia: further insights and recommendations for prevention of a clinically threatening artifact. Clin Chem Lab Med 2012;50:1281-1285.
4 von dem Borne AE, van der Lelie H, Vos JJ, van der Plasvan Dalen CM, Risseeuw-Bogaert NJ, Ticheler MD, Pegels HG: Antibodies against cryptantigens of platelets. Characterization and significance for the serologist. Curr Stud Hematol Blood Transfus 1986;:33-46.

5 Berkman N, Michaeli Y, Or R, Eldor A: EDTAdependent pseudothrombocytopenia: a clinical study of 18 patients and a review of the literature. Am J Hematol 1991;36:195-201.
Bizzaro N: EDTA-dependent pseudothrombocytopenia: a clinical and epidemiological study of 112 cases, with 10-year follow up. Am J Hematol 1995;50:103-109.

7 Cohen AM, Cycowitz Z, Mittelman M, Lewinski UH, Gardyn J: The incidence of pseudothrombocytopenia in automatic blood analyzer. Haematologia (Budap) 2000;30:117-121.

8 Zandecki M, Genevieve F, Gerard J, Gordon A: Spurious counts and spurious results on haematology analysers: a review. Part I: platelets. Int J Lab Hematol 2007;29:4-20. 
9 Silvestri F, Virgolini L, Savignano C, Zaja F, Velisig M, Baccarani M: Incidence and diagnosis of EDTAdependent pseudothrombocytopenia in a consecutive outpatient population referred for isolated thrombocytopenia. Vox Sang 1995;68:35-39.

10 Lau LG, Chng WJ, Liu TC: Transfusion medicine illustrated. Unnecessary transfusions due to pseudothrombocytopenia. Transfusion 2004;44:801.

11 Biner B, Devecioğlu O, Demir M: Pitfalls in the diagnosis of immune thrombocytopenic purpura in children: 4 case reports. Clin Appl Thromb Hemost 2007; 13:329-333.

-12 Yamada EJ, Souto AF, de Souza Eda E, Nunes CA, Dias $\mathrm{CP}$ : Pseudothrombocytopenia in a patient undergoing splenectomy of an accessory spleen. Case report. Rev Bras Anestesiol 2008;58:488-491, 485-488.

13 Sharma A, Pinto Pereira LM, Capildeo K, Charles K, Teelucksingh S: Steroid-induced iatrogenic disease after treating for pseudothrombocytopenia. Clin Appl Thromb Hemost 2011;17:100-102.
14 Yin XL, Shen WD, Chen YS, Zhou Y, Zhang XH: Refractory platelet transfusion in a patient with CD36 deficiency due to pseudothrombocytopenia. Platelets 2011;22:237-240.

15 Albersen A, Porcelijn L, Schilders J, Zuetenhorst H, Njo T, Hamberg P: Sunitinib-associated pseudothrombocytopenia induced by IgM antibody. Platelets 2013; 24:566-570.

16 Forscher CA, Sussman II, Friedman EW, Solomon V, Spaet TH: Pseudothrombocytopenia masking true thrombocytopenia. Am J Hematol 1985;18:313-317.

17 Schwarzinger I, Speiser W, Lubenow N, Greinacher A, Panzer S: Heparin-platelet factor (PF) 4 antibodies in patients with pseudothrombocytopenia: coincidence or association? Thromb Haemost 2000;84:1123-1124.

18 Zimrin AB, Warkentin TE: Transient pseudothrombocytopenia associated with immune heparin-induced thrombocytopenia complicated by pulmonary embolism. Thromb Haemost 2013;109:971-973.
19 Fozza C, Pardini S, Marras T, Longu F, Isoni A, Contini S, Longinotti M: Pseudothrombocytopenia in a patient receiving romiplostim for immune thrombocytopenic purpura. Ann Hematol 2014;93:899-900.

20 Allerheiligen D, Houston R, Vermedahl B: EDTA-induced pseudothrombocytopenia. J Am Board Fam Pract 1996;9:212-214.

-21 Lin J, Luo Y, Yao S, Yan M, Li J, Ouyang W, Kuang M: Discovery and correction of spurious low platelet counts due to EDTA-dependent pseudothrombocytopenia. J Clin Lab Anal 2014; doi: 10.1002/jcla.21818.

22 Nagler M, Keller P, Siegrist D, Alberio L: A case of EDTA-dependent pseudothrombocytopenia: simple recognition of an underdiagnosed and misleading phenomenon. BMC Clin Pathol 2014;14:19. 